\title{
Optimisation of Stellarator Equilibria with ROSE
}

\author{
M. Drevlak, C. D. Beidler, J. Geiger, P. Helander, Y. Turkin \\ Max-Planck-Institut für Plasmaphysik, IPP-EURATOM Association, Germany
}

\begin{abstract}
It is well known that optimisation of the MHD equilibrium of stellarators can have great influence on the performance and hence have a significant effect on the potential of stellarators as potential power plants.

The ROSE code was written to carry out an optimisation based on an equilibrium evaluation using VMEC and several additional codes. This paper presents both a quasiaxially symmetric and a quasi-isodynamic equilibrium obtained with ROSE and demonstrates two important features that are new to stellarator optimisation. One allows the reduction of coil complexity by analysing a current sheet obtained with NESCOIL. The second is a new way of accounting for properties of the vacuum field while simultaneously optimising the finite beta equilibrium.
\end{abstract}

\section{Introduction}

The properties and performance of stellarators depend sensitively on details of the underlying MHD equilibrium [1], which is (in the absence of magnetic islands) determined by the shape of the plasma boundary and by the current and pressure profiles [2]. For the last several decades, there have been stellarator design studies trying to find magnetic-field geometries with particularly favourable confinement and stability properties. In most such studies, the design of the MHD equilibrium is done in two stages. The shape of the plasma boundary is found in the first stage, and magnetic-field coils producing this plasma shape are sought in a second stage. There are several reasons for separating the optimisation of the coils from that of the plasma shape in this way. A practical reason is that fixed-boundary MHD equilibria are more easily and quickly computed than freeboundary ones. A more fundamental reason for first optimising the plasma shape is that the problem of seeking coils producing a given magnetic field is inherently ill-posed in the sense that there are in general many different sets of currents that create approximately the same magnetic field. In some respects, this is fortunate, since if it were not the case, the (unique) set of coils required for the desired field would in general be impracticable, perhaps impossible to build. It is precisely because the problem of finding magnetic-field coils for a given field is ill-posed that it is possible to find practical solutions. However, in practice these two stages of optimisation are usually not completely separated. For instance, the target function used when optimising the plasma shape may include some "guess" as to the coil complexity. Also in the design of the National Compact Stellarator Experiment (NCSX) at PPPL, the coil shapes were even directly included in the optimisation of the plasma shape [3].

Concerning the first stage, there are several physics goals of any optimisation. Historically, the first issue to receive attention was MHD stability. A notable example of a device optimised for this property is TJ-II [4], which has configurations that are stable up to very high values of $\langle\beta\rangle$, the volume average of the normalised plasma pressure. A number of further optimisation goals have also played a role. In the optimisation of 
Wendelstein 7-X (W7-X) [5], great emphasis was put on the minimisation of the PfirschSchlüter (PS) currents, which have the potential to alter the properties of the equilibrium and cause an outward (Shafranov) shift of the plasma column. The PS current can also play a role in MHD stability. If the PS current is minimised, one makes sure that the shape and position of the plasma is robust against finite-pressure effects. Likewise, the bootstrap (BS) current was minimised in order to reduce the influence of any internal currents on the equilibrium. The small currents in W7-X allow the use of an island divertor that relies on the rotational transform at the plasma edge being equal to one of the "natural" rational values $5 / 6,5 / 5$ or $5 / 4$. This need for a stable $\iota$-profile adds to the necessity to keep the (BS) current as small as possible. This is particularly important since the global magnetic shear is small, making the location of islands sensitive to small changes in the toroidal plasma current.

Finally, in stellarators with the ambition of relevance for power plant designs, it is important to demonstrate that fast ions can be well confined. Stellarators are not intrinsically able to confine trapped fast particles (see for instance [1]), and even the W7-X design exhibits difficulties in this regard, which may lead to severe heat loads on in-vessel components during neutral-beam injection [6]. In a reactor with W7-X geometry, there would be "hot spots" on the wall from escaping alpha particles. Several strategies have been employed to achieve good fast-particle confinement. One approach is to adjust the magnetic field so that the Fourier spectrum of $|B|$ exhibits a helical or toroidal symmetry in Boozer coordinates [7], a so-called quasi-symmetry. Examples of quasi-symmetric configurations are the HSX device [8] (quasi-helical symmetry) in Madison, Wisconsin, the CHS-qa [9] design study, and the NCSX effort [10,11], both with quasi-axial symmetry. W7-X, too, is a configuration optimised for enhanced fast particle confinement. It has drift surfaces that are highly aligned with the flux surfaces of the magnetic field, making the trapped particles precess poloidally in the region around the magnetic axis. Unfortunately, the optimisation is only effective at high plasma beta close to the magnetic axis - in practice in a region covering only a portion of the deposition profile of neutral-beam particles.

The present paper describes a new code, ROSE, which was written for the purpose of optimising MHD equilibria for stellarators. In addition to a detailed description of the code itself, two examples are presented to showcase the capabilities of the code in general and, in particular, to demonstrate the usefulness of two innovations ROSE introduces to stellarator optimisation.

The remainder of this paper is organised as follows: Section 2 gives a survey of the general structure and of the most important capabilities of the ROSE code. Sec. 3 explains in detail how ROSE computes the vacuum field of a finite $\langle\beta\rangle$ equilibrium solution. Sec. 4 discusses an example of an equilibrium optimised with respect to vacuum-field properties, and in Sec. 5 the approach for targeting coil complexity is explained. Sec. 6 finally describes an example of a configuration that was optimised for simple coils.

\section{The ROSE Code}

The purpose of the ROSE (ROSE Optimises Stellarator Equilibria) code is the optimisation of stellarator MHD equilibria. It provides a functionality that is similar to capabilities contained in the STELLOPT package [12], and software of similar charac- 
ter has also been reported in [13]. The code reuses large quantities of code from the ONSET [14] [15] and EXTENDER [16] packages.

A fixed-boundary VMEC MHD equilibrium [17] is defined by the shape of the plasma boundary and two flux functions, which are commonly chosen among the plasma pressure $p(s)$, the rotational transform $\iota(s)$ or the current density $I^{\prime}(s)$ as functions of the normalised toroidal magnetic flux $s=\Phi_{\text {tor }} / \Phi_{\text {tor }}^{\text {boundary }}$. $\Phi$ denotes the toroidal flux within some flux surface. The plasma boundary can be described by a pair of Fourier series:

$$
r(u, v)=\sum_{m, n} r_{m n} \cos (2 \pi(m u+n v)), \quad z(u, v)=\sum_{m, n} z_{m n} \sin (2 \pi(m u+n v))
$$

where $u$ and $v$ are poloidal and toroidal surface coordinates, respectively, chosen to have a period of unity, so that, e.g., $u$ varies from 0 to 1 over one poloidal revolution around the surface. The toroidal coordinate $v$ is related to the toroidal angle as $v=\frac{N_{p} \varphi}{2 \pi}$, where $N_{p}$ is the number of field periods and $\varphi$ is the true toroidal angle.

The ROSE code uses one of several optimising algorithms to minimise a target function

$$
F\left(\left\{r_{m n}, z_{m n}\right\}\right)=\sum_{i} w_{i}\left(f_{i}\left(r_{m n}, z_{m n}\right)-f_{i}^{\text {target }}\right)^{2} \sigma_{i}\left(f_{i}\right)
$$

in the parameter space $\left\{r_{m n}, z_{m n}\right\}$.

It is interesting to note that the parametrisation (1) of the boundary is not unique in the poloidal direction. As a consequence, it is probably possible to find a more compact representation of the plasma boundary that would lead to a reduced parameter set for the optimisation.

The functions $\sigma_{i}$ are $\sigma \equiv 1$ for properties that require a specific target value. An example would be the rotational transform, which usually is desired to have a specific target value. On the other hand, there can be $\sigma_{i}=0$ for $f_{i}<f_{i}^{\text {target }}$ or $f_{i}>f_{i}^{\text {target }}$ if $f_{i}$ is a function for which low or high values are desirable.

The coefficient $r_{0,0}$, in the following referred to as the major radius, is held constant during the optimisation. The same can be done for the aspect ratio, defined as

$$
A=\frac{r_{0,0}}{\sqrt{r_{1,0} z_{1,0}}} .
$$

ROSE has several optimising algorithms available. The most important one is the algorithm proposed by Brent [18], which is also amply used in the ONSET package, but there are in addition several other evolutionary algorithms. These include Particle Swarm [19], Differential Evolution [20], Harmony Search [21] and regular genetic optimisation [22]. The software structure for the evolutionary algorithms was inspired by Yedder [23].

The optimisation campaigns carried out in stellarator design operate on a complex, non-linear target function in a high-dimensional parameter space. Evidence for the existence of local optima has been observed. Therefore, it is beneficial to have a selection of different optimisation strategies available.

The target function $F\left(\left\{r_{m n}, z_{m n}\right\}\right)$ is computed along the following steps: 
1. Investigate the plasma boundary for viability. This step discards plasma boundaries that are considered pathological and so avoids VMEC calculations that have no chance of ever converging to a useful solution. To be specific, plasma boundaries are discarded if at any toroidal cross section the plasma boundary intersects itself or if the number of inflection points exceeds a given threshold.

2. Attempt a fixed-boundary VMEC calculation. This computational step establishes an equilibrium solution for a given plasma boundary parameterised by coefficients $\left\{r_{m n}, z_{m n}\right\}$. After this step (if successful) the magnetic field at the design value of $\langle\beta\rangle$ is known.

3. Attempt calculation of the $B_{m n}$ Fourier spectrum of the magnetic field strength in Boozer coordinates from the VMEC solution using the VM2MAG code [24].

4. If requested, the complexity of coils (yet to be designed) is assessed by using the codes SURFGEN and NESCOIL $[25,26]$. This step calculates the surface current on a surface $S$ exterior to the plasma that would create the required magnetic field inside the plasma. The surface $S$ should be chosen so as to approximate the surface on which the coils will lie in order to produce a reliable estimate of coil complexity which depends on the distance between plasma and coils. The result of the NESCOIL step is a continuous current distribution. If the latter is very complicated, the coils are likely to be complicated too. This step is new in stellarator equilibrium optimisation and will be explained further in Sec. 5 .

5. If requested, compute from the plasma currents the corresponding field components normal to the plasma surface, and use SURFGEN and NESCOIL to obtain the vacuum field underlying this equilibrium. This step is also new, and will be described in due detail in Sec. 3.

6. Use the data produced during the preceding steps to compute all relevant properties of the equilibrium. A detailed list of these items will be outlined below.

If any of the computational steps (1) to (3) in this sequence fails, the remainder of the sequence is aborted and the target function is set to a large value. No in-depth calculation of the detailed properties of the equilibrium is then carried out.

From the files computed during this sequence, individual properties are extracted in order to evaluate the partial target functions $f_{i}$. These properties include, for example:

- The integrated maximum absolute value among the principal curvatures (eigenvalues of the surface curvature tensor) of the plasma boundary surface: $\max \left\{\left|K_{1}\right|,\left|K_{2}\right|\right\}$.

- Integrated absolute value of the Gaussian curvature $|K|=\left|K_{1} K_{2}\right|$. Note the importance of taking the absolute value of $|K|$. The surface integral (no absolute value) of $\mathrm{K}$ yields a constant that depends on the topology of the surface only (Gauss-Bonnet theorem).

- Value of magnetic well, $\frac{\mathrm{d}^{2}}{\mathrm{~d} s^{2}} V$, at the design value of $\langle\beta\rangle$, where $V(s)$ denotes the volume of the magnetic surface enclosing the normalised toroidal flux $s$. The 
magnetic well can be calculated from the line integral $\int d l / B$, performed over many turns around the torus [27]. The magnetic well is of importance for MHD stability, which is discussed further-below.

- Total plasma volume, $V(1)$.

- Value of rotational transform $\iota(s)$ at selected radial positions $s$.

- Occurrence of regions with undesired sign of the global magnetic shear.

- Field ripple on the magnetic axis, defined in terms of the maximum and minimum field strengths

$$
\mathcal{R}=\frac{B_{\max }-B_{\min }}{B_{\max }+B_{\min }}
$$

This quantity is expected to be small for quasi-helically and quasi-axially symmetric stellarators.

- For quasi-isodynamic and for quasi-helically symmetric configurations, the mirror ratio

$$
\mathcal{M}=\frac{B(s, u=0, v=0)-B(s, u=0, v=1 / 2)}{B(s, u=0, v=0)+B(s, u=0, v=1 / 2)} .
$$

Quasi-helically symmetric and quasi-isodynamic configurations have extremal values of the magnetic field strength at the symmetry planes $v=0, v=1 / 2$. The mirror ratio is important for fast-particle confinement and for the bootstrap current, and can assume unfavourable magnitudes if not constrained.

- Deviation of the $B_{m n}$ spectrum (in Boozer components) from quasi-symmetry. Keeping this deviation small is important for fast-particle confinement in quasisymmetric configurations.

- Ratios between arbitrary $B_{m n}$ components. These ratios may be used to adjust a configuration for small bootstrp current.

- Effective helical ripple (as defined in Ref. [28]) on selected magnetic surfaces. This quantity can be used to target neoclassical confinement.

- Radial drift velocity parameters $\Gamma_{v}, \Gamma_{w}$ (as defined in Ref. [29]) on selected magnetic surfaces, as indicators of fast-particle confinement.

- The variance $\operatorname{var}(B)$ of the field strength on the contours of maximum and minimum $B$. These contours lie within flux surfaces and close toroidally in the case of a quasi-axially symmetric (QA) configuration, helically for a quasi-helically symmetric $(\mathrm{QH})$ configuration and poloidally for a quasi-isodynamic design. See Fig. 1 for illustration.

- Monotonicity of $|B|$ on a flux surface in a direction orthogonal to a symmetry direction. See Fig. 1 for illustration. This monotonicity of the field strength is important for the confinement of fast particles. 
- Variance of the second adiabatic invariant $J\left(B_{0}\right)=\int_{B(l)<B_{0}} \sqrt{B_{0}-B(l)} d l$ on a flux surface. The code computes $J$ for the confinement region of adjacent field lines covering the entire unit square in $u, v$. The variance is taken from the set of $J$ values obtained.

- Properties involving the vacuum field:

- Vacuum rotational transform on the axis

- Vacuum magnetic well

- Shafranov shift, defined as the horizontal distance between the magnetic axes in the vacuum field and that at the design value of $\langle\beta\rangle$.

- Properties related to coil complexity, see Sec. 5 for a detailed discussion.

- Average orbit loss rates, with the drift orbits calculated along the lines of Ref. [30].

The items related to the curvatures in this list require additional clarification. The curvature of the plasma boundary is not in itself a good indicator of the suitability of the final configuration. Nevertheless, it is useful when an optimisation "from scratch" is attempted. In this case, meaningful configurations are usually so distant in parameter space from the staring point that the optimisers tested in the framewoek of ROSE fail to find a path to a viable stellarator, and instead get lost in poor local optima often characterised by strong shaping of the boundary. Imposing a penalty on strong curvatures helps the optimisation to avoid these local minima. The penalty for the curvature should be dropped at an intermediate stage of the campaign and eventually be replaced by penalties targeting coil complexity.

\section{Optimisation for Properties of the Vacuum Field}

For many configuration properties, it is desirable to carry out the optimisation at finite plasma pressure. This is particularly the case for qualities related to fast-particle confinement, the accuracy of quasi-symmetries, or the effective ripple. These properties depend on the plasma pressure, and it is their value at finite $\langle\beta\rangle$ that is decisive for the performance of the stellarator in question. However, important properties of the vacuum field can become difficult to discern or assume unfavourable values. For instance, due to the diamagnetic property of the plasma, the magnetic well can be positive [corresponding to negative $V^{\prime \prime}(s)$ ] at finite $\langle\beta\rangle$ but turn into a magnetic hill $\left(V^{\prime \prime}>0\right)$ when the vacuum field is analysed. Similar considerations apply to the rotational transform $\iota(s)$. In lowshear configurations, magnetic islands can be avoided by tailoring the $\iota$-profile to avoid low-order rational numbers, but one must then take care to ensure that this property is not lost as $\langle\beta\rangle$ is varied. In order to be able to address these difficulties, ROSE has been given the ability to compute the vacuum magnetic field directly from the VMEC solution.

Analyses of vacuum field properties have been done before in the scope of the NCSX design effort [31]. There, the vacuum field was evaluated using a virtual casing (VC) directly on the plasma boundary. The drawback of this approach is that the VC field close to the plasma boundary becomes subject to a severe discretisation error if the $\mathrm{VC}$ 


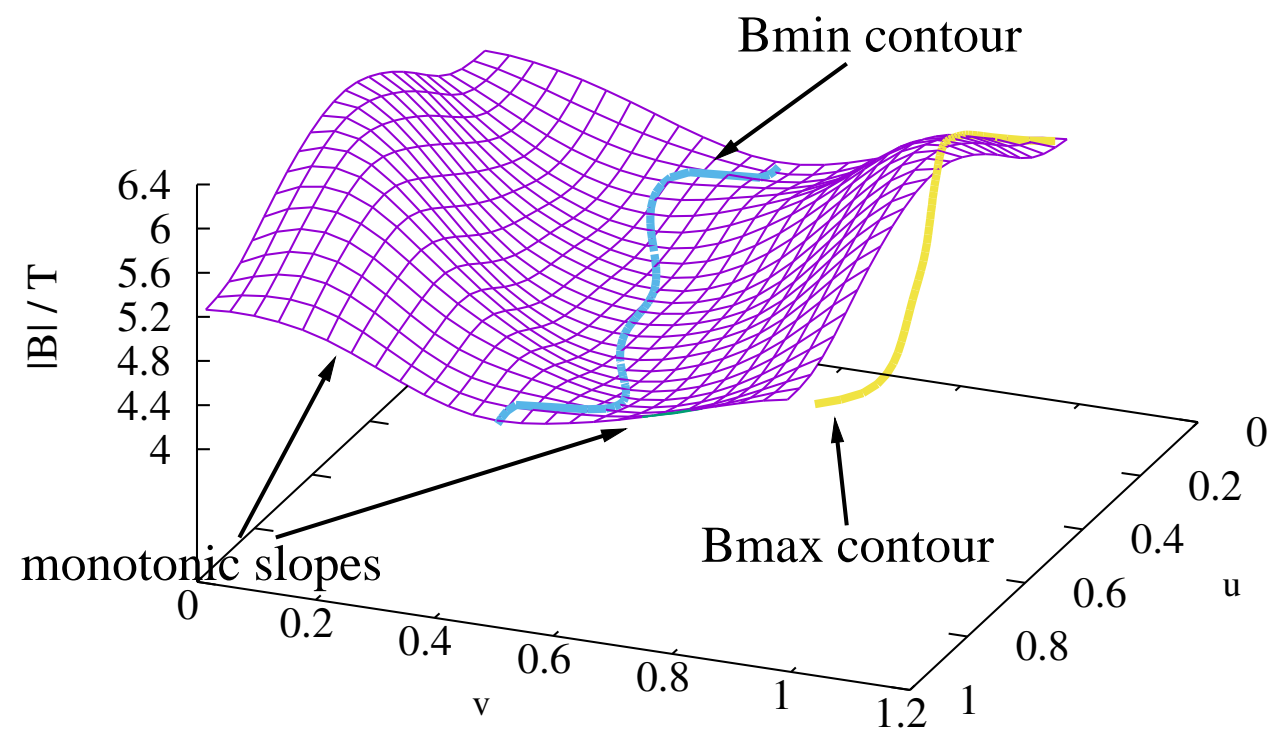

Figure 1: Structure of the magnetic field in the case of W7-X. Shown is the magnitude of the magnetic field and the location of the extremal contours of the magnetic field. The slopes between high and low field regions are monotonic. 
current (Biot Savart) is integrated on a grid with fixed mesh size. If the Biot-Savart integration is done adaptively, any desired degree of accuracy can be maintained but the computational effort increases. Moreover, no vacuum field estimate beyond the plasma boundary is possible.

The approach chosen for ROSE proceeds along three major steps as follows:

1. calculate the normal magnetic field from plasma currents on the plasma boundary

2. compute a current sheet generating the true vacuum field using SURFGEN and NESCOIL

3. evaluate vacuum field properties using the vacuum field computed from the current sheet using the Biot-Savart formula

The details of these steps will be outlined in the following sections.

\subsection{Calculating the normal magnetic field}

The naive approach for obtaining the vacuum field would be to just rerun VMEC (in fixedboundary mode) with the same boundary but with vanishing plasma pressure. However, this approach has a number of serious shortcomings:

1. It is inaccurate because it assumes identical plasma boundaries at vacuum and finite $\langle\beta\rangle$. In reality, the two are different, and especially for cases with large PS currents this difference may be significant.

2. It is slow because it requires another full VMEC computation.

For these reasons, an approach was chosen that relies on the virtual-casing principle to obtain the normal field from plasma currents or external coils exactly on the plasma boundary, and then carrying out a NESCOIL run to compute the field in the entire plasma domain.

To explain this procedure in greater detail, let us divide $\quad \mathbb{R}^{3}$ into 2 disjoint domains $V_{1}$ and $V_{2}$, with current distributions $\mathbf{J}_{1}$ and $\mathbf{J}_{2}$ in each of these. The current densities $\mathbf{J}_{1}$ and $\mathbf{J}_{2}$ generate contributions $\mathbf{B}_{1}$ and $\mathbf{B}_{2}$ to the magnetic field so that $\mathbf{B}_{1}(x)+$ $\mathbf{B}_{2}(x)=\mathbf{B}(x)$ Assume, finally, that the boundary $\delta V$ between $V_{1}$ and $V_{2}$ is located so that $\mathbf{B} \cdot \mathbf{n}=0$.

If $V_{1}, V_{2}$ are chosen as the domains inside and outside the plasma boundary, then $\mathbf{B}_{1}$ and $\mathbf{B}_{2}$ are the magnetic field contributions from inner and outer currents respectively. $\mathbf{B} \cdot \mathbf{n}=0$ on the plasma boundary implies that $\mathbf{B}_{1} \cdot \mathbf{n}$ and $\mathbf{B}_{2} \cdot \mathbf{n}$ have the same magnitude.

In the following, let $\mathbf{p}(u, v)$ or $\tilde{\mathbf{p}}(u, v)$ denote points on the boundary surface and $\mathbf{p}_{u}=\frac{\mathrm{d} \mathbf{p}}{\mathrm{d} u}, \mathbf{p}_{v}=\frac{\mathrm{d} \mathbf{p}}{\mathrm{d} v}$, so that $\mathbf{n}=\frac{\left(\mathbf{p}_{u} \times \mathbf{p}_{v}\right)}{\left\|\mathbf{p}_{u} \times \mathbf{p}_{v}\right\|}$ denotes the normalised normal vector. The virtual-casing principle [32] as stated by Shafranov and Zakharov states that a image current density on $\delta V$ defined by

$$
\mathbf{J}_{\text {image }}(\mathbf{p})=-\mathbf{B}(\mathbf{p}) \times \mathbf{n}(\mathbf{p})
$$


creates a magnetic field,

$$
\mathbf{B}_{\text {image }}(\mathbf{x})=\frac{\mu_{0}}{4 \pi} \int_{\delta V} \frac{\mathbf{J}(\tilde{\mathbf{p}}) \times(\mathbf{x}-\tilde{\mathbf{p}}) \mathrm{d} \mathbf{A}}{\|\mathbf{x}-\tilde{\mathbf{p}}\|^{3}}
$$

such that

$$
\mathbf{B}_{\text {image }}(\mathbf{x})=\left\{\begin{array}{cc}
\mathbf{B}_{2}(\mathbf{x}) & \mathbf{x} \in V_{1} \\
-\mathbf{B}_{1}(\mathbf{x}) & \mathbf{x} \in V_{2}
\end{array}\right.
$$

For a mathematically rigorous treatment of the virtual-casing principle the reader is encouraged to consult Ref. [33].

It should be noted that there are extensions of the virtual-casing principle that drop the requirement of $n \cdot B=0$ and introduce monopole sources in order to deal with the normal field component [16]. In such a case, the magnetic field on the boundary would be discontinuous. In the context of the application considered here, however, the condition $\mathbf{B} \cdot \mathbf{n}=0$ implies that the normal field on the plasma boundary from the interior currents and those from exterior currents are identical except for an opposing sign. Therefore, while the tangential magnetic field created by the image current sheet is discontinuous on $\delta V$, the normal field is well-defined.

The evaluation of equation (7) needs to deal with a singularity in the Biot-Savart rule and requires a little sleight of hand. A similar calculation has been implemented in the BNORM code by Merkel [34]. The calculation starts by seeking the Biot-Savart integral for the vector potential:

$$
\mathbf{A}_{\text {image }}(\mathbf{p})=\frac{\mu_{0}}{4 \pi} \int_{0}^{N_{p}} \int_{0}^{1} \frac{\mathbf{J}_{\text {image }}(\tilde{\mathbf{p}})\left\|\tilde{\mathbf{p}}_{u} \times \tilde{\mathbf{p}}_{v}\right\|}{\|\mathbf{p}-\tilde{\mathbf{p}}\|} \mathrm{d} u \mathrm{~d} v
$$

An explicit encounter with the singularity is avoided by computing $\mathbf{A}_{\text {image }}(p)$ on integer points $\mathbf{p}\left(i / N_{u}, N_{p} j / N_{v}\right) \quad\left(N_{u}, N_{v}\right.$ denoting the number of mesh lines in the angular directions) using an open Newton-Cotes formula evaluating the integrand at half-integer points $\tilde{\mathbf{p}}\left(\left(i+\frac{1}{2}\right) / N_{u}, N_{p}\left(j+\frac{1}{2}\right) / N_{v}\right)$. The code also allows a refined mesh for the Newton-Cotes integration using more than one point, spaced equidistantly between the integer mesh points, for the integration.

In addition to using the dual mesh, the numerical integration is simplified (and hence accelerated) by eliminating the singular part from the integrand. This is achieved by writing

$$
\begin{gathered}
\mathbf{A}_{\text {image }}(\mathbf{p})=\frac{\mu_{0}}{4 \pi} \int_{v-N_{p} / 2}^{v+N_{p} / 2} \int_{u-1 / 2}^{u+1 / 2}\left[\frac{\mathbf{J}_{\text {image }}(\tilde{\mathbf{p}})\left\|\tilde{\mathbf{p}}_{u} \times \tilde{\mathbf{p}}_{v}\right\|}{\|\mathbf{p}-\tilde{\mathbf{p}}\|}\right. \\
\left.-\frac{\mathbf{J}_{\text {image }}(\mathbf{p})\left\|\mathbf{p}_{u} \times \mathbf{p}_{v}\right\|}{\sqrt{\hat{u}^{2} \mathbf{p}_{u}^{2}(u, v)+2 \hat{u} \hat{v} \mathbf{p}_{u}(u, v) \mathbf{p}_{v}(u, v)+\hat{v}^{2} \mathbf{p}_{v}^{2}(u, v)}}\right] \mathrm{d} \tilde{u} \mathrm{~d} \tilde{v} \\
+\frac{\mu_{0}}{4 \pi} \int_{v-N_{p} / 2}^{v+N_{p} / 2} \int_{u-1 / 2}^{u+1 / 2} \frac{\mathbf{J}_{\text {image }}(\mathbf{p})\left\|\mathbf{p}_{u} \times \mathbf{p}_{v}\right\|}{\sqrt{\hat{u}^{2} \mathbf{p}_{u}^{2}(u, v)+2 \hat{u} \hat{v} \mathbf{p}_{u}(u, v) \mathbf{p}_{v}(u, v)+\hat{v}^{2} \mathbf{p}_{v}^{2}(u, v)}} \mathrm{d} \tilde{u} \mathrm{~d} \tilde{v}
\end{gathered}
$$

with $\hat{u}=\tilde{u}-u, \hat{v}=\tilde{v}-v$ 
The first of these integrals has the singularity removed and yields directly to numerical integration. The second part of (9) can be integrated analytically in one of the coordinates according to

$$
\begin{gathered}
\int_{-N_{p} / 2}^{N_{p} / 2} \int_{-1 / 2}^{1 / 2} \frac{\mathrm{d} \hat{u} \mathrm{~d} \hat{v}}{\sqrt{\hat{u}^{2} \mathbf{p}_{u}^{2}(u, v)+2 \hat{u} \hat{v} \mathbf{p}_{u}(u, v) \mathbf{p}_{u}(u, v)+\hat{v}^{2} \mathbf{p}_{v}^{2}(u, v)}}= \\
\frac{1}{\sqrt{p_{u}^{2}}} \int_{-N_{p} / 2}^{N_{p} / 2} \ln \frac{2 \sqrt{p_{u}^{2}} \sqrt{\frac{1}{4} \mathbf{p}_{u}^{2}+\mathbf{p}_{u} \mathbf{p}_{v} v+\mathbf{p}_{v}^{2} v^{2}}+\mathbf{p}_{u}^{2}+2 \mathbf{p}_{u} \mathbf{p}_{v} v}{2 \sqrt{\mathbf{p}_{u}^{2}} \sqrt{\frac{1}{4} \mathbf{p}_{u}^{2}-\mathbf{p}_{u} \mathbf{p}_{v} v+\mathbf{p}_{v}^{2} v^{2}}+\mathbf{p}_{u}^{2}-2 \mathbf{p}_{u} \mathbf{p}_{v} v} \mathrm{~d} \hat{v} \\
=\frac{1}{\left\|\mathbf{p}_{u}(u, v)\right\|} \int_{-N_{p} / 2}^{N_{p} / 2} L(\hat{v}) \mathrm{d} \hat{v}
\end{gathered}
$$

Again, this integral can be split into a non-singular part and a singular one by adding and subtracting a singularity of the same type:

$$
\int L(\Delta v) \mathrm{d} \Delta v=\int\left(L(\hat{v})-\ln \frac{\mathbf{p}_{u}^{2}}{\mathbf{p}_{v}^{2} \hat{v}^{2}}\right) \mathrm{d} \hat{v}+\int \ln \frac{\mathbf{p}_{u}^{2}}{\mathbf{p}_{v}^{2} \hat{v}^{2}} \mathrm{~d} \hat{v}
$$

The first integral is readily evaluated numerically using a Gauss-Kronrod rule while the second is calculated analytically.

Once $\mathbf{A}_{\text {image }}$ has been computed on the integer mesh on the plasma boundary, the tangential components are interpolated using a $2 \mathrm{~d}$ spline with periodic boundary conditions. The normal component $\mathbf{n} \cdot \mathbf{B}_{\text {plasma }}$ is then obtained by taking the normal component of curl $\mathbf{A}_{\text {image }}$, i.e. from the tangential derivatives of the tangential components of $\mathbf{A}_{\text {image }}$.

Note that the method outlined assumes that the tangential magnetic field is continuous at the plasma boundary. This neccessitates a continuous pressure profile, so the pressure must go to zero.

Moreover, strict rigour also requires the rotational transform on the plasma boundary to be irrational to prevent the presence of magnetic islands [36] [37]. The distinction between regions with closed flux surfaces, islands and chaotic field lines is beyond the computational scope of the VMEC code.

\subsection{Calculating the external field}

Once the normal magnetic field from the plasma currents on the plasma boundary is available, a NESCOIL run is performed that is corrected for the normal magnetic field.

Generally, NESCOIL computes a current distribution on a given toroidal currentcarrying surface (CCS) so as to minimise the normal magnetic field on another, inner, surface. The latter usually is a desired plasma boundary, and minimising the normal field on that surface leads to a field that has a flux surface at this location.

The numerical approach used by NESCOIL assumes a current distribution on the CCS described by

$$
\mathbf{J}=\mathbf{n} \times \nabla \Phi, \quad \Phi=c_{u} u+c_{v} v+\sum_{k, l} s_{k l} \sin (2 \pi(k u+l v))
$$


where $n$ now denotes the unit normal vector to this surface. NESCOIL then uses a least-squares method to solve for the coefficients $s_{k l}$ so that

$$
\int\left(\mathbf{n} \cdot \mathbf{B}_{N E S C}+\mathbf{n} \cdot \mathbf{B}_{\text {plasma }}\right) \mathrm{d} \mathbf{A} \stackrel{!}{=} \min .
$$

This correction of the current sheet for the normal field from plasma currents was originally proposed and applied in the context of coil design for devices with large plasma currents [38].

The coefficients $c_{u}, c_{v}$ are fixed and describe the total toroidal and poloidal currents supplied by the coil set. For the applications treated in this context, it is consistently assumed that the configuration uses a modular coil set. This means that $c_{u}=0$ while $c_{v}$ must be matched to the poloidal current underlying the optimised equilibrium.

After the coefficients $s_{k l}$ of the current potential have been computed, the current density is computed on a mesh on the current surface. The magnetic vacuum field is then obtained directly from a discrete Biot-Savart formula on that current sheet. The current sheet is not cut into discrete coils since such an approach would not result in any computational benefit but add the difficulty of creating and handling coils of different topological nature. For instance, if the current potential contains local extrema, the corresponding equipotential lines would constitute local islands on the CCS that do not close poloidally. This would then result in the appearance of saddle coils among the "desired" modular coils. Fig 2 shows an example of a current potential and the associated current lines on a current surface located $60 \mathrm{~cm}$ from the plasma boundary for the configuration discussed in Sec. 6. Note that this current potential was computed at an elevated distance from the plasma boundary in order to illustrate different types of current lines. A CCS closer to the plasma will result in a much more benign current distribution.

Unlike the NESCOIL calculation used to assess the coil complexity (see Sec. 5), the current sheet for the vacuum field is not located in a region where the coils are expected, but in a region that is favourable for a benign NESCOIL solution and an efficient and accurate calculation of the Biot-Savart law. This is usually significantly closer to the plasma boundary than the coil set is.

\section{Example: HELIAS}

Our first example of an optimisation using ROSE is a HELIAS-type (i.e. W7-X-like) configuration aiming at improved confinement of trapped fast particles compared with W7-X. Specifically, the aim was to improve the confinement of such particles away from the magnetic axis whilst, at the same time, keeping the bootstrap current low, in order to facilitate an island divertor of the type used in W7-X. In the original design of W7-X, particle confinement was optimised at a quarter minor radius $(s=1 / 16)$, leaving the confinement at larger radii insufficient for a future reactor.

In order to improve the situation, a configuration was sought that exhibits minimal effective ripple $\epsilon_{\text {eff }}$ over most of the radial range $s$ while keeping the magnetic mirror, measured by $\mathcal{M}(5)$, within the range $10 \%<\mathcal{M}<15 \%$. The optimisation is carried out at a plasma pressure of $\langle\beta\rangle \sim 5 \%$ since it is at this pressure that a reactor would need to exhibit its best confinement properties. As in the case of W7-X, a configuration with $N_{P}=5$ periods was chosen, but the aspect ratio was set to $A=12.2$, which is slightly 


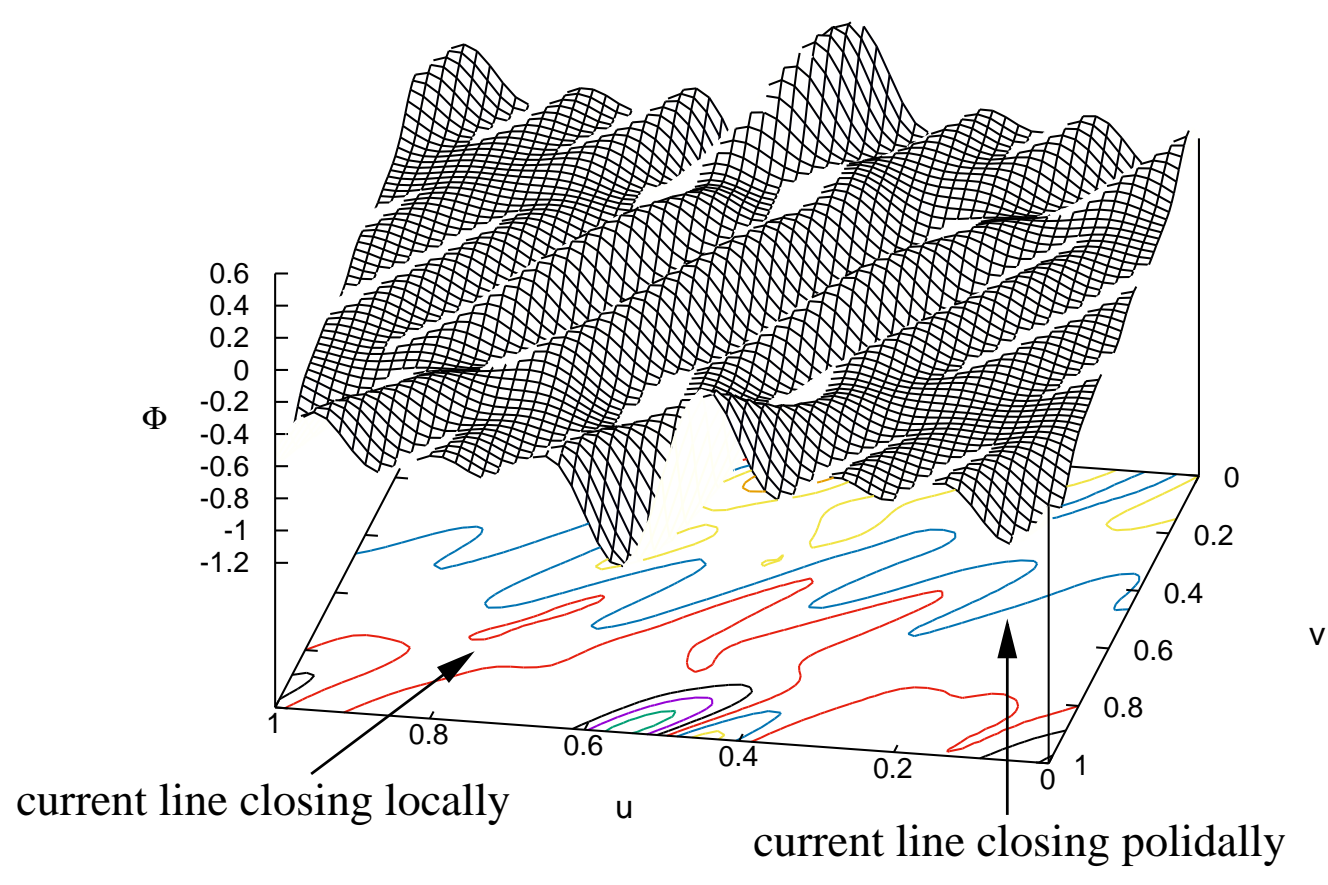

Figure 2: Example current potential for a quasi-axially symmetric configuration. The current flows alongh the contour lines of $\Phi$. Poloidally closed contour lines currespond to regular modular coils while locally closed contour lines imply the presence of saddle coils. This current potential was calculated at an elevated distance from the plasma boundary in order to illustrate the different possible types of current lines. 


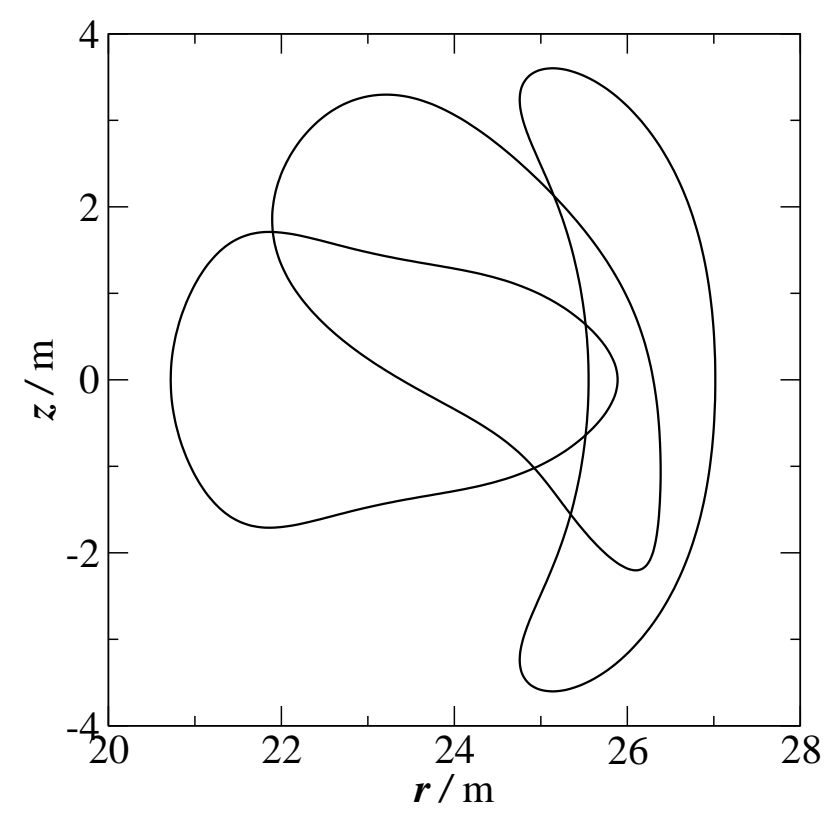

Figure 3: Toroidal cross sections of the optimised equilibrium

larger than in W7-X. This choice was motivated by the need for having enough room for a blanket and neutron shielding in a reactor [35]. For the rotational transform a profile approaching unity at the plasma boundary was targeted so as to facilitate the use of an island divertor like in W7-X. $\iota=0.98$ at $s=0.97$ was explicitly included in the target function.

The optimisation at finite $\langle\beta\rangle$ makes it difficult to ensure the existence of a vacuum magnetic well. For the finite- $\beta$ field, the existence of a well, created by the plasma currents, is virtually certain. However, its magnitude allows no reliable estimate of the existence and magnitude of any well in the vacuum field. Therefore, the vacuum well as outlined in Sec. 3 was explicitly included in the optimisation.

Fig. 3 shows a set of toroidal cuts of the plasma boundary of the VMEC solution of the optimised equilibrium. It can be seen that the plasma shape retains the general features seen in other optimised configurations [4] [8] [5]. The pronounced bean-like shape and the triangular cross section at the opposing symmetry plane are typical of configurations exhibiting a vacuum magnetic well.

Fig. 4 compares the magnetic well of the vacuum field and that at finite $\langle\beta\rangle$. The finite- $\langle\beta\rangle$ field has a specific magnetic volume that decreases rapidly in the radial direction, corresponding to a positive well. The magnetic well of the vacuum field is much smaller, but remains positive.

In Fig. 5 a comparison of the effective ripple between W7-X and the new HELIAS configuration is shown. The W7-X configuration used here is an early reference design [39], which has a mirror term of $\mathcal{M} \sim 13 \%$ directly built into the equilibrium. It can be seen that by directly targeting $\epsilon_{\text {eff }}$ in the optimisation a further reduction of neoclassical transport relative to $\mathrm{W} 7-\mathrm{X}$ is possible. For future optimisations it is expected that an effective ripple up to $\sim 1 \%$ will be sufficient.

Fig. 6 shows a plot of the magnetic field strength on the flux surface at $s=1 / 2$. 


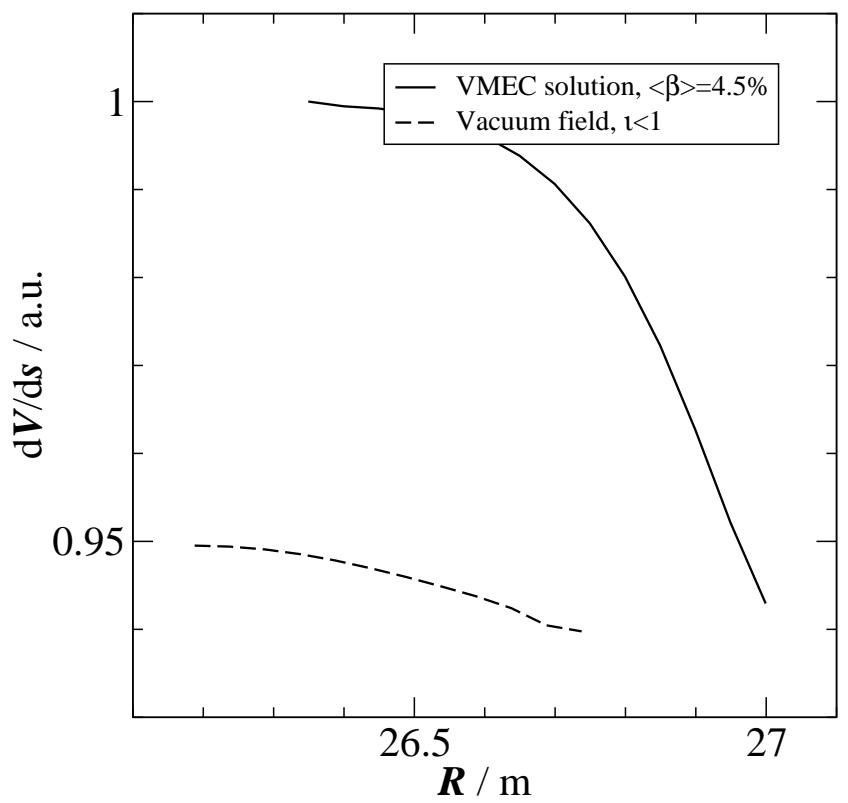

Figure 4: Specific magnetic volume for field lines traced in a $3 \mathrm{~d}$ calculation. $R$ is the radial cylindrical coordinate of the starting point.

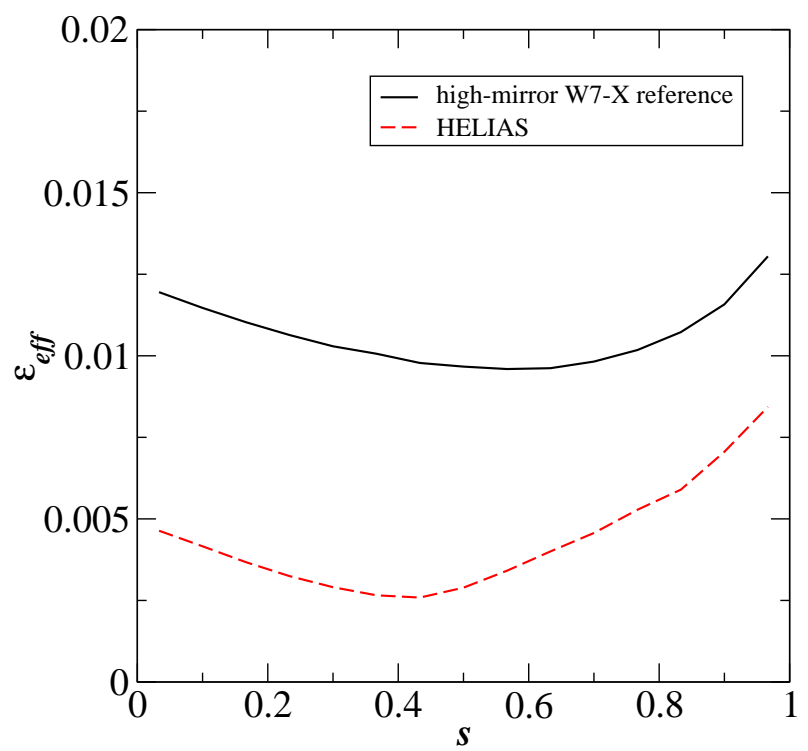

Figure 5: Effective ripple as a function of the normalised flux $s$. 


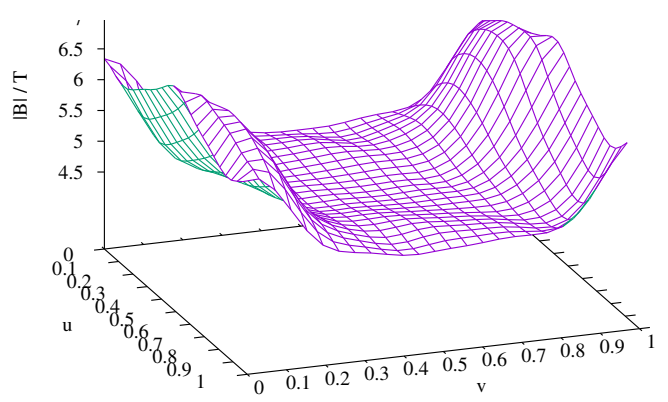

Figure 6: Absolute value of the magnetic field strength $|\mathbf{B}|$ as a function of surfaces coordinates $u, v$ on the flux surface located at $s=1 / 2$.

The field structure resembles the one found in [6], where the coil currents of W7-X were adjusted so as to optimise the confinement of fast particles. Specifically, the plot exhibits a flat valley of low field around the triangular symmetry plane at $v=1 / 2$, surrounded by a peaked maximum in the bean-shaped symmetry plane $(v=0 / v=1)$. While it is conceivable that this field structure would have been found using optimisation criteria more closely linked to fast particle confinement, this optimisation campaign explicitly sought to minimise the variation $\operatorname{var}(B)$ in the regions of largest and smallest field strength whilst

keeping $|B|$ monotonic in the toroidal direction between these regions.

The losses of fusion $\alpha$-particles of the new configuration are compared with those of the high-mirror reference W7-X equilibrium. The calculations were carried out using the ANTS code [6]. The equilibria were scaled up to reactor size $\left(1900 \mathrm{~m}^{3}, B=5 \mathrm{~T}\right)$ and the $\alpha$-particles were launched on individiual flux surfaces and with a realistic pitch angle distribution. The particle losses were calculated without collisions. The comparison between the two cases is shown in Fig. 7 .

Generally, it can be seen that for the new configuration the particle losses are reduced compared with the reference W7-X equilibrium. Only for particles launched at $s \geq 0.8$ is the confinement somewhat lower than in W7-X. This is of little relevance since the production of $\alpha$-particles in this region is expected to be small.

The temporal evolution of the particle losses highlights the existence of two different loss channels. Rapid losses are observed for times of typically $1 \mathrm{~ms}$. These losses are caused by deeply trapped particles. The rapid losses are followed by a slow rise of the loss fraction, sometimes accelerating at $\mathrm{t}=0.01 \mathrm{~s} \ldots 0.1 \mathrm{~s}$. These slow losses are primarily caused by barely reflected particles. The comparison sows that especially this class of losses is significantly reduced in the new equilibrium compared with W7-X.

Finally, it deserves to be pointed out that the reduction of fast-particle losses is strongest in regions with $s \leq 0.6$. This is desirable as it is expected that in this region the production of $\alpha$-particles will be highest. Nevertheless, it would be interesting if, as a result of future optimisations, losses of particles born at greater radii could be reduced, too. 


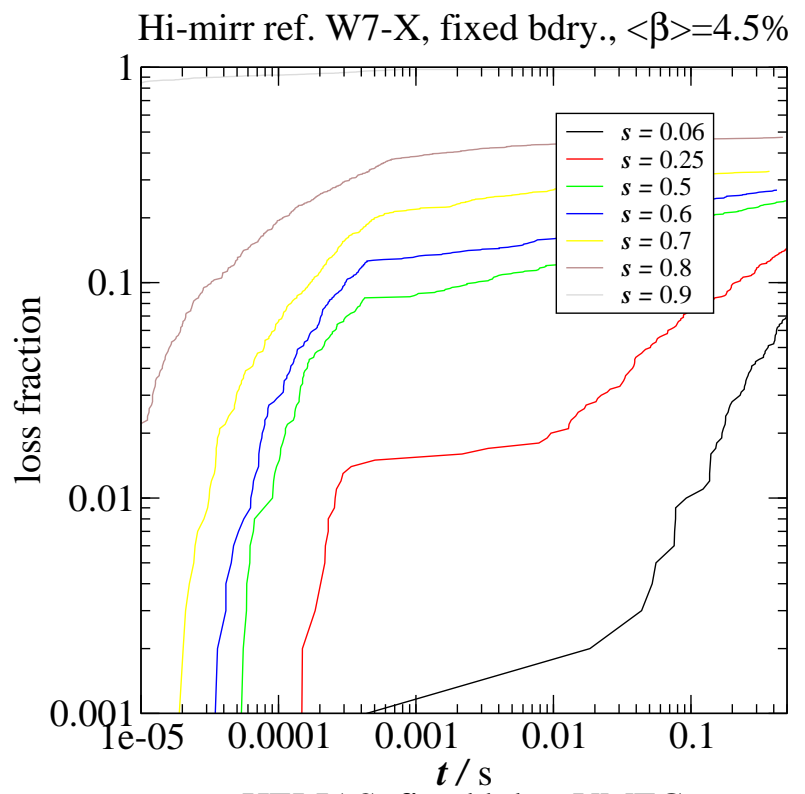

HELIAS, fixed bdry. VMEC

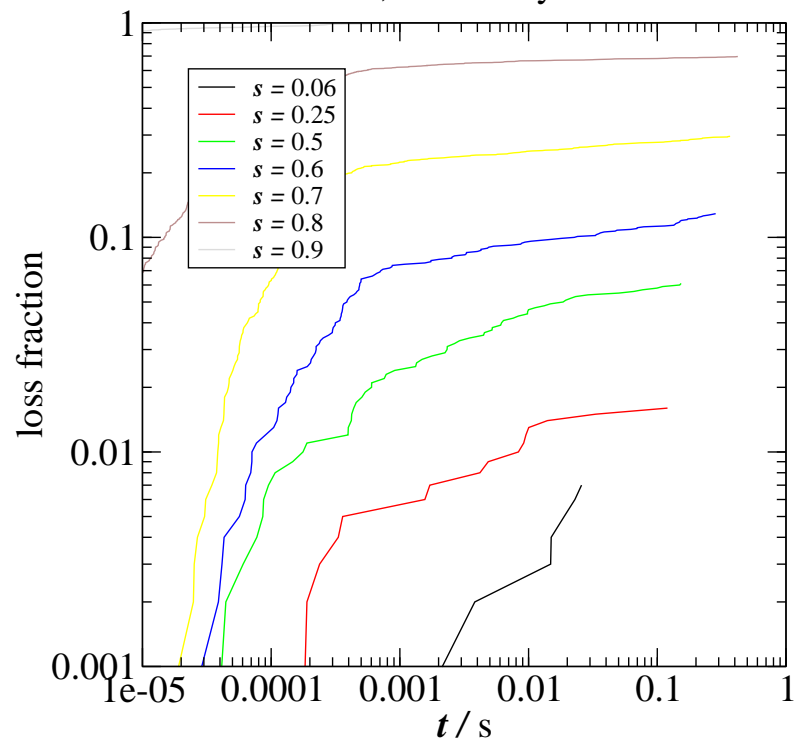

Figure 7: Collisionless losses of fusion $\alpha$-particles for the original high-mirror W7-X design and for the new HELIAS configuration. 


\section{Optimisation for Simple Coils}

Coil complexity is an issue with the potential of deciding the technological feasibility of a stellarator configuration. In W7-X, for example, the curvature radii of the central filament of the coils are, in some places, comparable to the lateral dimension of the coil casing. Curvature radii equal to or smaller than this value would pose a serious obstacle to the construction of a device. Similar considerations apply to inhomogeneities of the current distribution required to create a given magnetic configuration. If this current distribution peaks too strongly in individual locations, the resulting clearances between coil filaments could become too small to accommodate a finite-size coil cross-section.

Much effort has been made to address this difficulty. Coil optimisation tools like ONSET, COILOPT [40], FOCUS [41] [42] or REGCOIL [43] are able to seek coil sets with optimised curvature radii and filament clearances. However, the optimisation of these properties may impair other peoperties of the resulting magnetic field. Boozer and $\mathrm{Ku}$ have proposed methods to include the response of relevant plasma properties to slight modifications of the configuration in order to extend the potential for easing coil construction in directions that do not compromise plasma performance [44] [45] [46].

Questions related to finite coils (actual coil filaments as opposed to a continuous current distribution) have also been addressed in the past in the scope of the NCSX design, albeit with a different intention [47] [3]. There, a set of finite coils was included in the optimisation loop in order to address issues related to flux surface integrity. The resulting computational process was an integrated optimisation of coils and equilibrium. While this approach offers a very comprehensive optimisation scope, the computational effort is enhanced as an optimisation including free boundary equilibrium calculations is extended over the parameter space of a coil set, which usually is considerably larger than that of an equilibrium.

We now turn our attention to a new way of reducing coil complexity, with the aim of doing so not only at the coil design stage when the MHD equilibrium has already been selected, but to consider coil simplicity already during the design of the equilibrium. This can be achieved without actually having to design the coils simultaneously with the MHD equilbrium, but by adding adequate indicators for coil complexity to the equilibrium target function. A good "guess" for coil complexity can be obtained from NESCOIL by computing the current sheet on a current-carrying surface (CCS) located at a specified distance from the plasma boundary. In coil optimisation, the distance between plasma and coils usually is a compromise between two factors. On the one hand, the ability of the coil set to impose field components with high Fourier mode numbers on the plasma is reduced as the distance is increased. On the other hand, the modular ripple caused by discrete coils gets worse when the coils are located closer to the plasma. This means that coil complexity, and also the nature of this complexity, in general depends on the number of independent coil types that are allowed. A design with more coil types will result in a smaller modular ripple and hence in coils located more closely to the plasma boundary. This, in turn, implies smaller coil curvatures but, at the same time, smaller clearances between coils.

In the design of a power plant, sufficient space between coils and plasma must be provided to accommodate a breeding blanket and neutron shielding. In practice, this imposes a limit on the minimum clearance between coils and plasma exceeding one metre. 
Because of these considerations, when evaluating coil complexity as described above, the clearance between this CCS and the plasma boundary should be chosen to be similar to the distance expected in the final machine design. The resulting approach to assessing coil complexity proceeds along the following steps:

1. Use SURFGEN to generate a suitable current-carrying surface. This surface should have a shape resembling that of the plasma boundary and be situated at roughly constant distance from it. Experience shows that the current distribution found with NESCOIL then becomes simpler when the shape of the current surface conforms with the shape of the plasma boundary, compared with a surface for which simply the $r_{10}, z_{10}$ coefficients are increased.

2. Use NESCOIL to compute the current distribution $J_{c}$ on the current surface.

3. Assess the complexity of this current distribution by evaluating the following properties:

- Harmonic content of the current potential. This quantity, defined by

$$
\mathcal{H}=\sqrt{\frac{\sum_{k, l} s_{k l}^{2}\left(k^{2}+l^{2}\right)}{c_{u}^{2}+c_{v}^{2}}},
$$

establishes a measure of the higher-order mode content of the current distribution required to reproduce a plasma equilibrium.

- Current compression. Strong peaks in the current density correspond to locations in the coil set where the clearance between central filaments is particularly small. In order to obtain an indication of these density peaks, the current compression is defined as

$$
\mathcal{C}=\frac{\max \left(J_{c}\right)-\min \left(J_{c}\right)}{\max \left(J_{c}\right)+\min \left(J_{c}\right)}
$$

- Geodesic and 3d curvature. The geodesic and 3d curvature, $\kappa_{g} a n d \kappa$, of the current lines are evaluated from the first and second derivatives of the surface point $(r(u, v), z(u, v))$ and of the current potential $\Phi(u, v)$ with respect to the surface coordinates $u, v$. Using these curvatures, the following quantities are computed as an indicator of coil curvature:

$$
\mathcal{K}=R_{\text {maj }} \sqrt{\frac{\int_{A} \kappa^{2} J_{c}^{2} \mathrm{~d} A}{I^{2}}}
$$

and

$$
\mathcal{K}_{g}=R_{\text {maj }} \sqrt{\frac{\int_{A} \kappa_{g}^{2} J_{c}^{2} \mathrm{~d} A}{I^{2}}}
$$

where $R_{m a j}$ is the major radius of the configuration.

The normalising factor

$$
I=\sqrt{\left(I_{\text {tor }}^{2}+I_{\text {pol }}^{2}\right)}
$$


represents the total magnitude of the external currents. In this implementation, $I_{p o l}$ is the external poloidal current (coils) in one machine period. Likewise, $I_{t o r}$ is the external toroidal current.

- Current islands. In a modular coil set, all current lines of the current sheet should close poloidally. However, the current potential may exhibit local extrema which lead to current lines that close locally around the respective extremum instead of poloidally. These sets of locally closed current lines are referred to as "current islands". If such a current sheet is discretised to form coils, these current islands would translate into saddle coils. if the construction of a modular coil set is intended, the presence of islands in the current sheet is undesirable.

The detection of current islands is carried out by investigating the current potential for local extrema. These can be detected by seeking locations where the gradient $\nabla_{u, v} \Phi(u, v)$ of the current potential becomes opposite to the regular gradient established by the total external current $\left(c_{u}, c_{v}\right)$.

$$
\begin{gathered}
\mathcal{I}=\frac{1}{c_{u}^{2}+c_{v}^{2}} \iint T\left(-\left(\nabla_{u, v} \Phi(u, v) \cdot\left(\begin{array}{c}
c_{u} \\
c_{v}
\end{array}\right)\right)+\gamma_{t}\right) \mathrm{d} u \mathrm{~d} v \\
T(x)=\left\{\begin{array}{cc}
0 & x<0 \\
x & \text { else }
\end{array}\right.
\end{gathered}
$$

The parameter $\gamma_{t}$ is a threshold parameter that facilitates detection and suppression of marginal current islands or helps the optimiser avoid that the current distribution starts developing island-like features.

\section{Example: ESTELL}

In this section a quasi-axially symmetric stellarator is presented that was explicitly designed for easy construction, suitable for a university experiment $[48,49]$. The intention is not to design a device located on a scaling path leading to a viable fusion power plant, but rather to propose a configuration suitable for basic plasma research, including an exploration of the properties of QA configurations. The configuration is characterised by the following basic parameters:

- major radius $R_{m a j}=1.4 m$

- $N_{p}=2$ machine periods

- rotational transform $1 / 5<\iota(s)<1 / 4$

- aspect ratio $A \sim 5.3$

- plasma volume $V=1.9 \mathrm{~m}$

- $B=0.5 \mathrm{~T}$

- $\langle\beta\rangle \sim 0.5 \%$ 
In order to arrive at a solution with simple coils, the criteria outlined in Sec. 5 were included in the target function for the equilibrium.

The optimisation was carried out at $\langle\beta\rangle=0$ as is it expected the device will be equipped with little heating power. As $\beta$ increases, almost any QA configuration will exhibit an appreciable bootstrap current, which will exert a considerable influence on the equilibrium. In a final design of this device, these effects will have to be addressed by additional coils, in particular a set of PF coils to counteract the outward Shafranov shift of the plasma. This design step is, however, beyond the scope of this paper and is not important for, or directly related to, the general methodology presented here. The Fourier coefficients of the optimised plasma boundary are listed in Table 1.

Figure 8 shows a set of cross sections of the equilibrium at toroidal coordinates $v=$ $0 / 0.25 / 0.5$. It can be seen that the stellarator shaping of this configuration is modest, which is essentially due to the relatively small rotational transform per period.

Fig. 9 shows the $\iota$-profile of the configuration at $\langle\beta\rangle=0$. The resonance at $\iota=2 / 10$ is avoided.

Fig. 10 shows the Fourier spectrum in Boozer coordinates of the magnetic field strength. It can be seen that the configuration approximates quasi-axisymmetry with very good accuracy. The largest non-QA component only reaches a value of $B_{2,1} \sim 2 \%$ at the plasma boundary and drops, like all $B_{m n}$ except for $B_{1,0}$, to a very small level inside the plasma.

After the optimised equilibrium had been produced, a coil design was carried out using the ONSET suite of codes as described in the previous section. A design with 6 coil types was chosen since in Ref. [49] it had been shown that, for a very similar design, the effective ripple improves noticeably up to 6 coil types while showing only little improvement beyond 6 coils. Fig. 11 shows a view of the coil set obtained. The maximum curvature of any central filament is $\kappa=4.15 \mathrm{~m}^{-1}$, which is modest for a machine of this size. The smallest clearance between any two filaments is $d \sim 13.6 \mathrm{~cm}$.

Fig. 12 compares the effective ripple of the fixed-boundary equilibrium obtained from the optimisation with that of the field produced by the coil set. It can be seen that $\epsilon_{\text {eff }}$ for finite coils is typically a factor 2 larger than for the fixed-boundary VMEC solution. Nevertheless, it remains small in comparison with the effective ripple reported for many other stellarator configurations [50].

\section{Conclusions}

Stellarator designs viable for a power plant need to be refined beyond the state of many current devices. The methods presented in this paper address two issues that are of practical relevance for the required optimisation procedures. The simultaneous optimisation of vacuum and finite- $\langle\beta\rangle$ fields yields an efficient way to keep track of the behaviour of the configuration under different plasma conditions. More importantly, the vacuum magnetic well offers a proxy for Mercier stability of the configuration.

The inclusion of coil complexity addresses one of the most important difficulties in the design of stellarators. Targeting this aspect early during the design of the equilibrium allows the optimiser the most comprehensive degree of information while seeking a favourable balance between coils complexity and other parameters. 


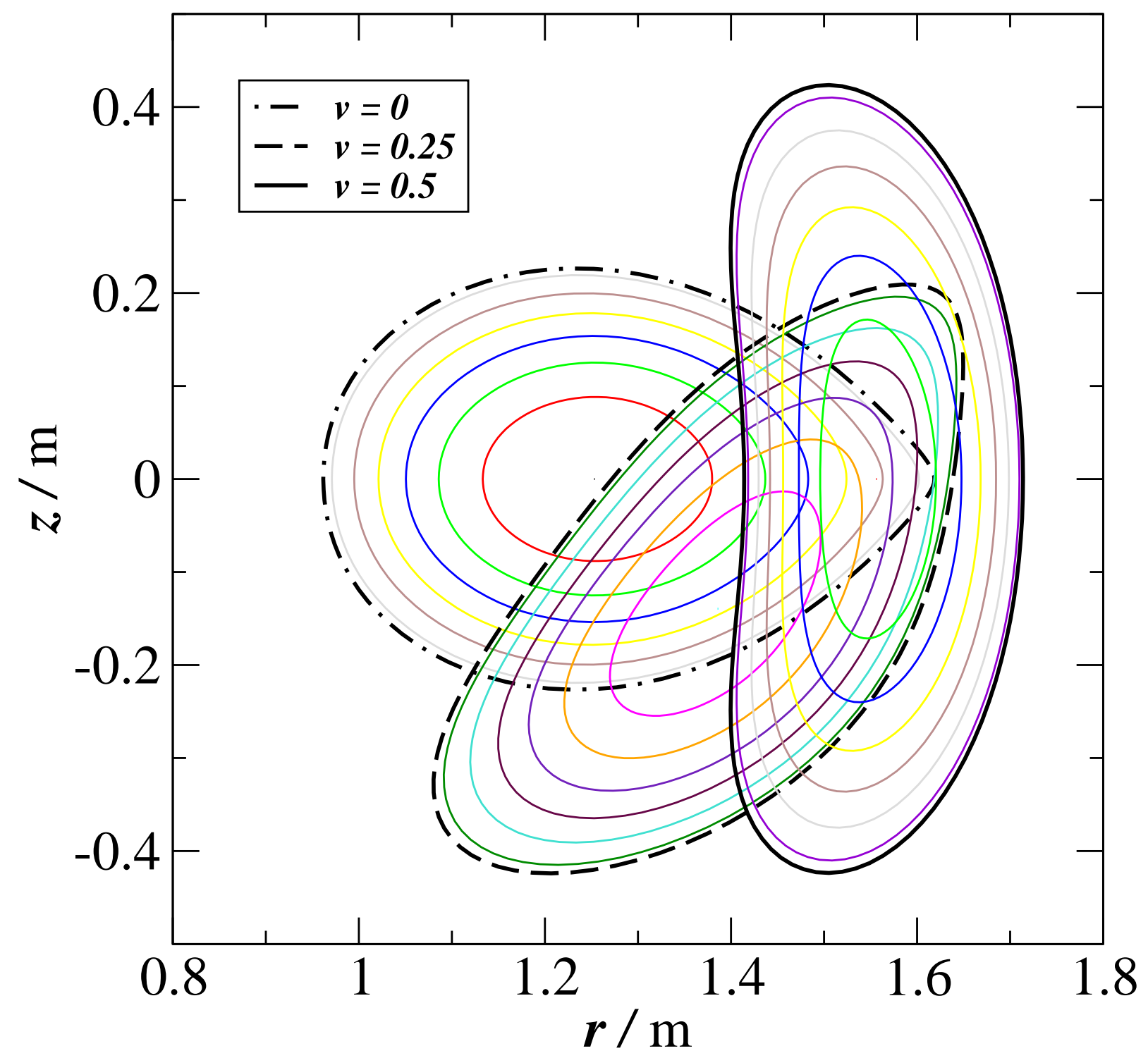

Figure 8: Toroidal cuts of the plasma boundary at $v=0 / 0.25 / 0.5$ 


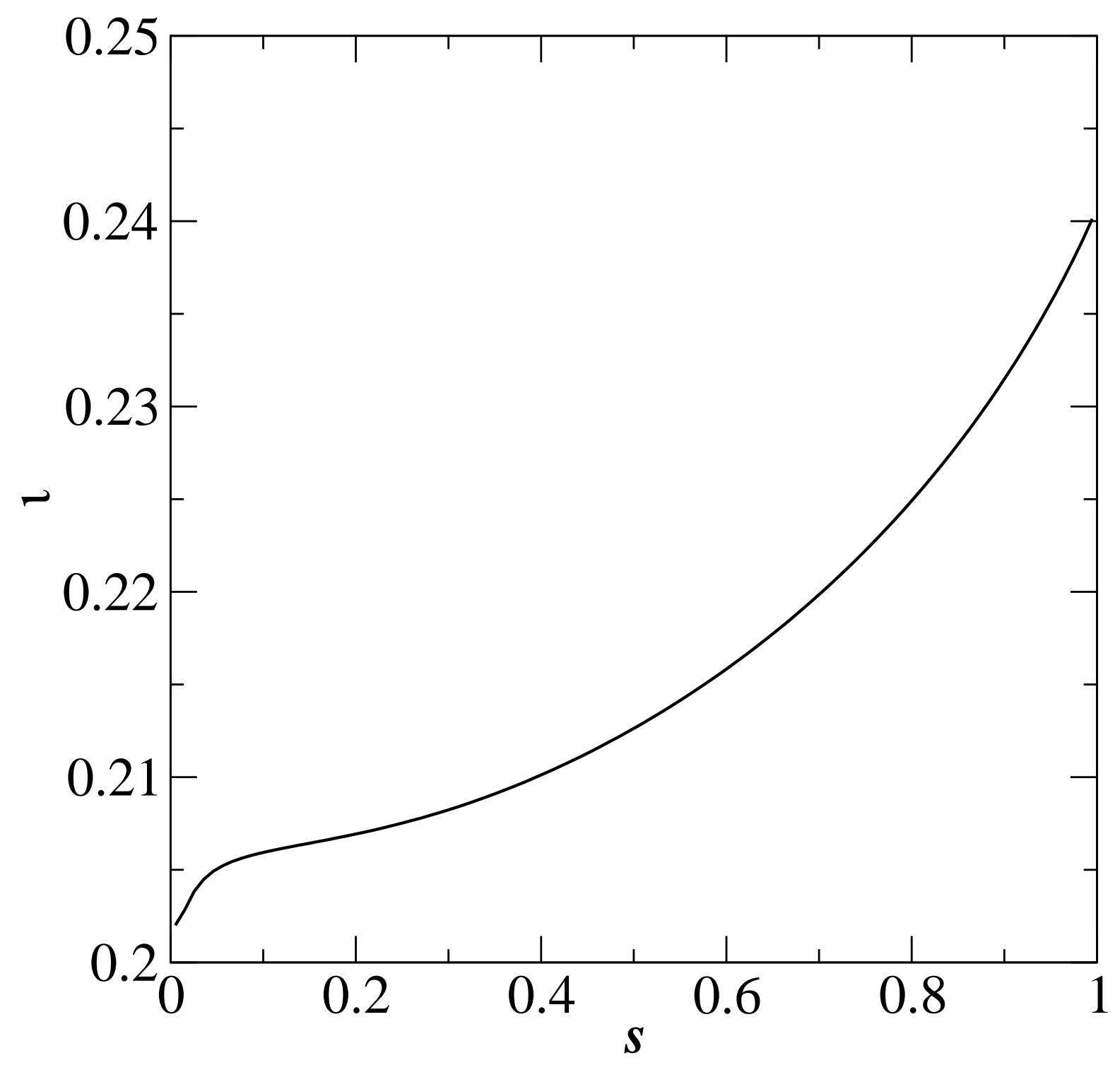

Figure 9: Rotational transform at $\langle\beta\rangle=0$ : 


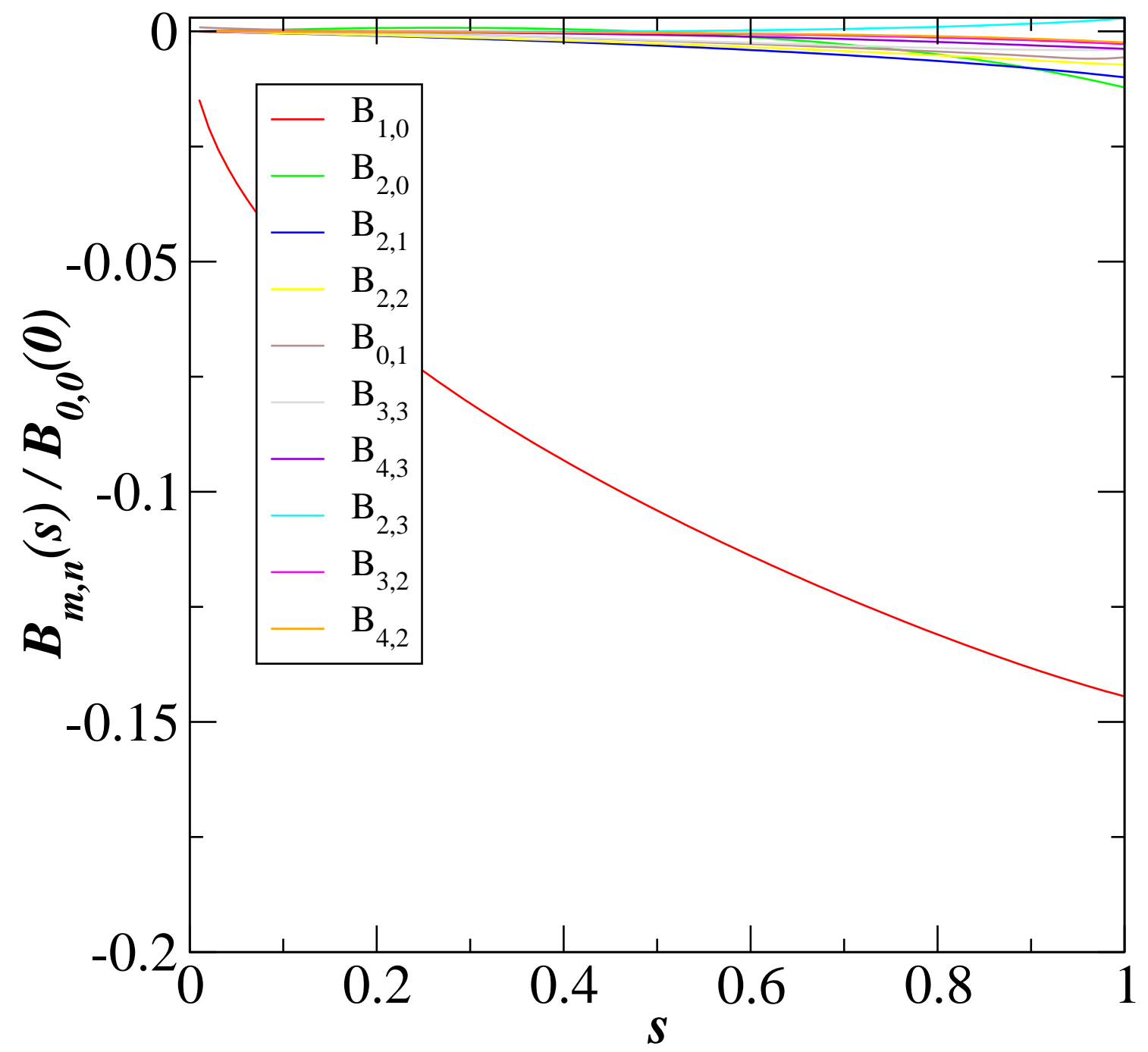

Figure 10: Radial dependence of the most important modes of $B_{m n}(s) / B_{00}(0)$ of the optimised QA configuration. 


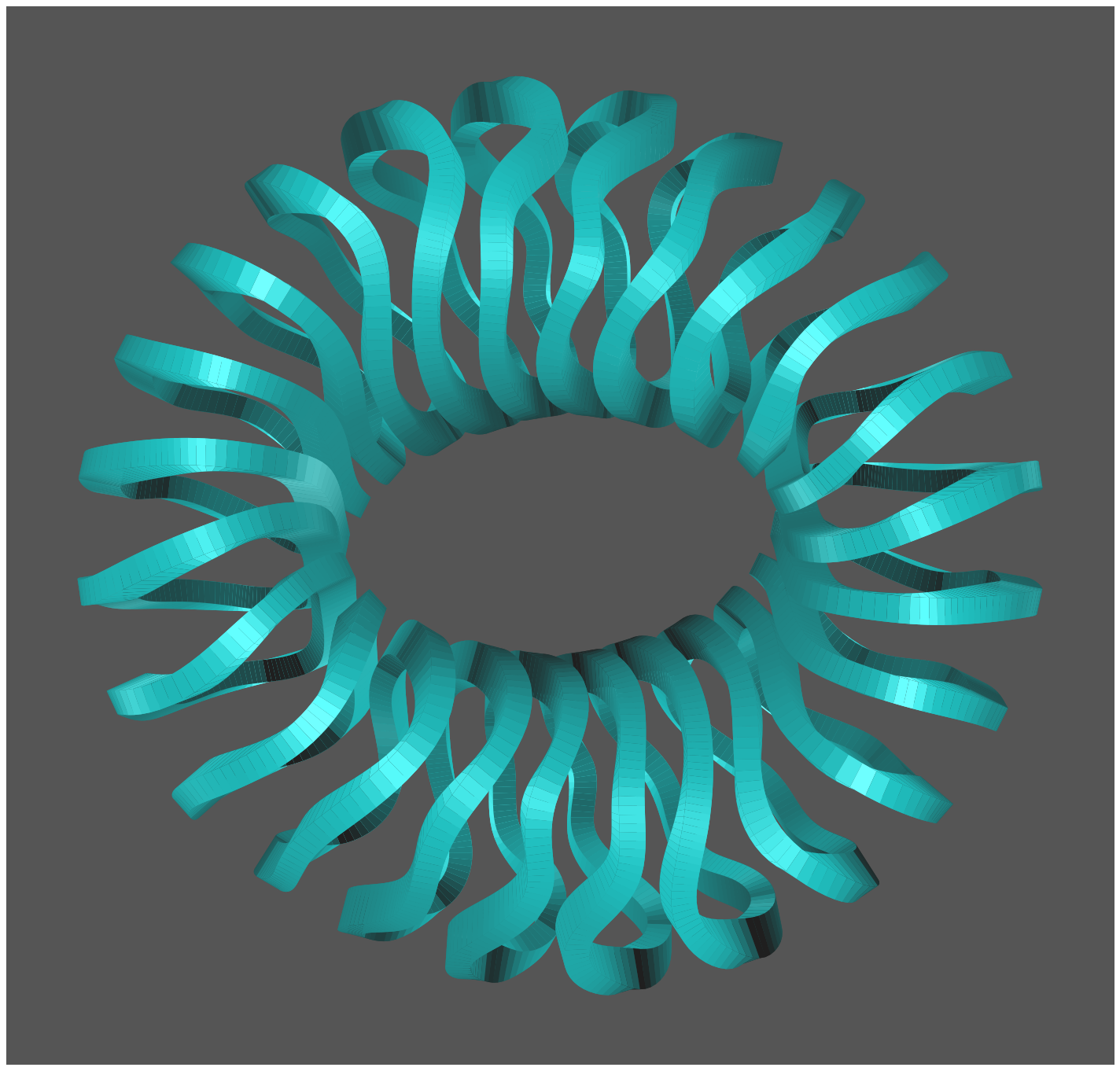

Figure 11: Birds eye view of the optimised coil set. 


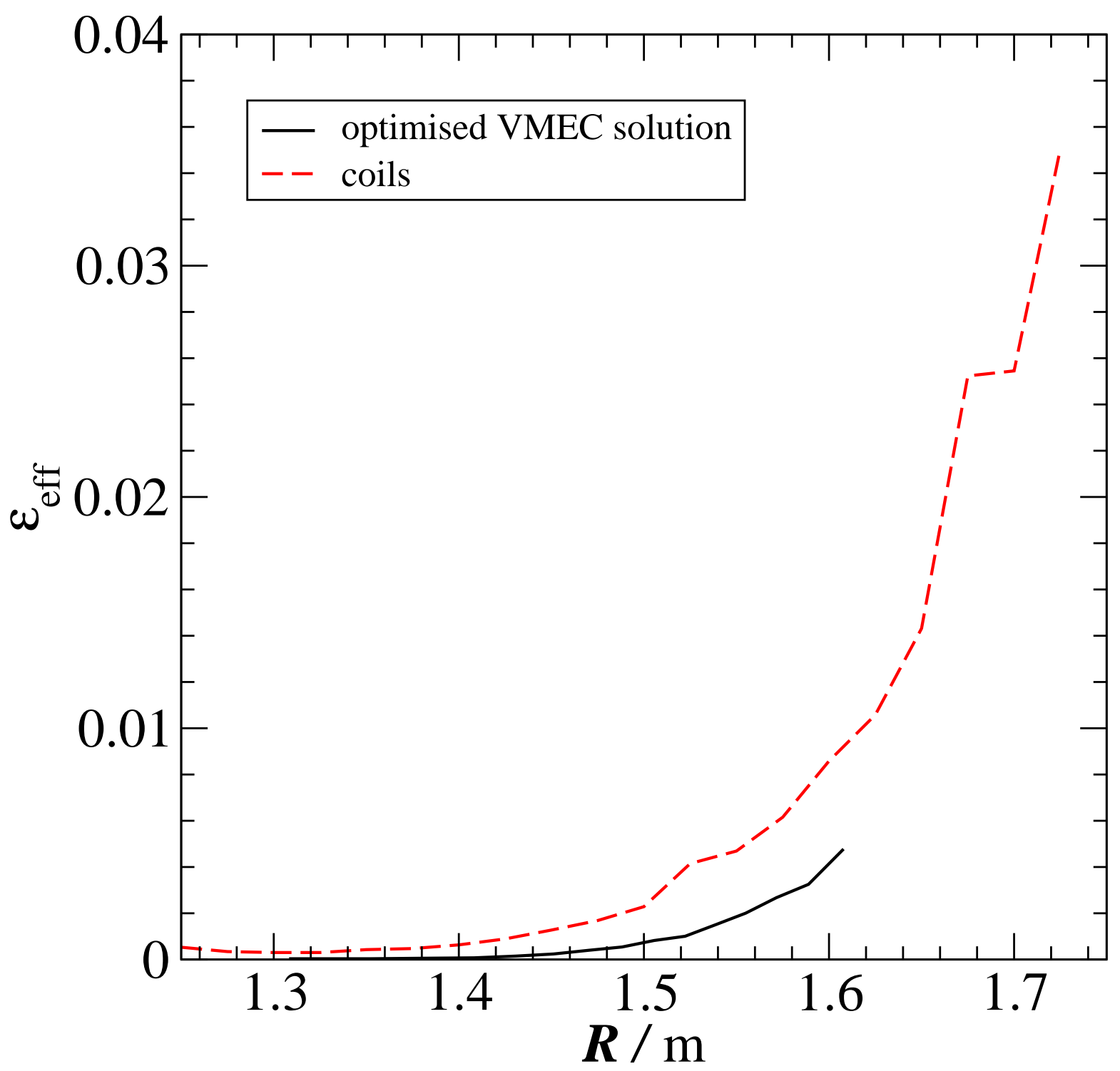

Figure 12: Profile of the effective ripple $\epsilon_{e f f}$ for the design plasma configuration () and for the optimised coil set (). Note that $\epsilon_{e f f}$ has been plotted over the radius of the staring point of the field lines instead of the flux label $s$. The vacuum field generated by the coils exhibits good flux surfaces and finite values of $\epsilon_{\text {eff }}$ beyond the $2 / 5$ islands visible as a flat region at $R<1.7 \mathrm{~m}$. 


\begin{tabular}{|rr|r|r|}
\hline$m$ & $n$ & $r_{m n} / \mathrm{m}$ & $z_{m n} / \mathrm{m}$ \\
\hline 0 & 0 & $1.4 \mathrm{e}+00$ & 0.0 \\
0 & -1 & $-1.2545 \mathrm{e}-01$ & $9.4567 \mathrm{e}-02$ \\
0 & -2 & $8.3293 \mathrm{e}-03$ & $-1.3073 \mathrm{e}-03$ \\
0 & -3 & $-9.7246 \mathrm{e}-05$ & $5.5650 \mathrm{e}-04$ \\
0 & -4 & $-6.5443 \mathrm{e}-05$ & $5.9706 \mathrm{e}-05$ \\
1 & 2 & $-8.1374 \mathrm{e}-05$ & $2.6490 \mathrm{e}-05$ \\
1 & 1 & $-1.0004 \mathrm{e}-02$ & $-4.3852 \mathrm{e}-03$ \\
1 & 0 & $2.6098 \mathrm{e}-01$ & $2.9895 \mathrm{e}-01$ \\
1 & -1 & $9.8954 \mathrm{e}-02$ & $-9.9384 \mathrm{e}-02$ \\
1 & -2 & $-4.1320 \mathrm{e}-03$ & $3.6010 \mathrm{e}-03$ \\
1 & -3 & $-7.8717 \mathrm{e}-04$ & $1.4219 \mathrm{e}-04$ \\
1 & -4 & $-9.5298 \mathrm{e}-06$ & $-5.9671 \mathrm{e}-06$ \\
2 & 3 & $-3.2841 \mathrm{e}-06$ & $-1.2213 \mathrm{e}-06$ \\
2 & 2 & $-2.5256 \mathrm{e}-03$ & $-3.8920 \mathrm{e}-03$ \\
2 & 1 & $6.6229 \mathrm{e}-03$ & $7.2629 \mathrm{e}-03$ \\
2 & 0 & $1.0945 \mathrm{e}-02$ & $-3.1021 \mathrm{e}-03$ \\
2 & -1 & $-2.1751 \mathrm{e}-02$ & $-1.5862 \mathrm{e}-02$ \\
2 & -2 & $9.6671 \mathrm{e}-03$ & $-8.5811 \mathrm{e}-03$ \\
2 & -3 & $3.7590 \mathrm{e}-03$ & $-2.3013 \mathrm{e}-03$ \\
2 & -4 & $-1.0702 \mathrm{e}-04$ & $3.4083 \mathrm{e}-04$ \\
3 & 1 & $-2.2935 \mathrm{e}-04$ & $1.0566 \mathrm{e}-04$ \\
3 & 0 & $-1.7837 \mathrm{e}-02$ & $-1.3451 \mathrm{e}-02$ \\
3 & -1 & $-4.9713 \mathrm{e}-04$ & $-1.3688 \mathrm{e}-03$ \\
3 & -2 & $2.0095 \mathrm{e}-04$ & $-7.7560 \mathrm{e}-03$ \\
3 & -3 & $1.8512 \mathrm{e}-03$ & $-2.5299 \mathrm{e}-03$ \\
4 & -2 & $8.5552 \mathrm{e}-04$ & $-1.3627 \mathrm{e}-03$ \\
\hline
\end{tabular}

Table 1: Fourier coefficients of the plasma boundary of ESTELL. 
The code, ROSE, used to implement these improvements and to carry out the optimisations requires further extensions to be able to carry out more comprehensive optimisations. The immediate goal is the addition of a full MHD stability analysis, including a ballooning analysis, to the target function. It should however be noted that linear MHD stability is perhaps not strictly necessary for good plasma performance in stellarators. There are plenty of experimental results suggesting that other optimisation criteria may be more important [1].

The configurations presented above, finally, need to receive a more comprehensive optimisation. This holds in particular for the QI configuration for which. in addition to improved fast-particle confinement, the complexity of the coil set needs to be included. Finally, quasi-helically symmetric configurations deserve a through exploration.

\section{Acknowledgements}

The authors thank M. Mikhailov, H. Maaßberg, and J. Nührenberg for useful discussions and support.

This work has been carried out within the framework of the EUROfusion Consortium and has received funding from the Euratom research and training programme 2014-2018 under grant agreement No 633053. The views and opinions expressed herein do not necessarily reflect those of the European Commission. 


\section{References}

[1] M. Hirsch et al., "Major results from the stellarator Wendelstein 7-AS" Plasma Phys. Control. Fusion (2008) 50053001

[2] M. D. Kruskal and R. M. Kulsrud "Equilibrium of a Magnetically Confined Plasma in a Toroid" Phys. Fluids 1 (1958) pp. 265

[3] A. Reimann et al., "Equilibrium and Flux Surface Issues in the Design of NCSX", Fusion Science and Technology 51 (2007) 145

[4] C. Alejaldre, "TJ-II Project: A Flexible Heliac Stellarator", Fusion Technol 17131 (1990)

[5] J.Nührenberg, R. Zille "Stable stellarators with medium $\beta$ and aspect ratio" Physics Letters A, Volume 114A, No. 3 (1986) pp. 129

[6] M. Drevlak et al., "Fast particle confinement with optimized coil currents in the Wr-X stellarator" Nuclear Fusion 54 (2014) 073002

[7] A. H. Boozer, "Transport and isomorphic equilibria" Phys. Fluids 26 (1983) pp. 496

[8] A. F. Almagri et al., "Design and construction of HSX: a helically symmetric stellarator", J. Plasma Fusion Res. SERIES, Vol.1 (1998) 422-425

[9] K. Matsuoka et al., "Post-CHS Project", Plasma Physics Reports 23 No. 7 (1997), pp. 588

[10] G. H. Neilson et al., "Physics issues in the design of High-beta, low-aspect-ratio stellarator experiments", Phys. Plasmas 7 No. 5 (2000) pp. 1911

[11] G. H. Neilson et al., "Physics optimization of stellarators", Phys. Fluids B 4 No. 7 (1992) pp. 2081

[12] D. A. Spong et al., "Physics issues of compact drift optimized stellarators" Nucl. Fusion 41 (2001) 711

[13] F. Castejon et al., "Stellarator optimization under several criteria using metaheuristics", Plasma Phys. Control. Fusion 55 (2013) 014003

[14] M. Drevlak, "Coil Designs for a Quasi-Axially Symmetric Stellarator", 20th Symposium on Fusion Technology (1998), Marseille, pp. 883

[15] "Optimisation of Heterogenous Magnet Systems", M. Drevlak, 12th International Stellarator Workshop, Madison, Wisconsin (1999)

[16] M. Drevlak et al., "Pies free boundary stellarator equilibria with improved initial conditions" Nucl. Fusion 45 (2005) pp. 731 
[17] S.P.Hirshman, van Rij, P.Merkel, "Three-dimensional free boundary calculations using a spectral Greens function method" Comput. Phys. Commun. 43, 143 (1986)

[18] R. P. Brent, "Algorithms for Minimization without Derivatives" Prentice-Hall, 1973, Ch. 5

[19] K. E. Parsopoulos, M. N. Vrahatis "Recent Approaches to Global Optimization Problems through Particle Swarm Optimization", Natural Computing 1 (2002) p. 235-306

[20] R. Storn, K. Price, "Differential evolution: A Simple and Efficient Heuristic for Global Optimization over Continuous Spaces", Journal of Global Optimization 11 (1997) pp. 341

[21] Kang Seok Leea, Zong Woo Geemb "A new meta-heuristic algorithm for continuous engineering optimization: harmony search theory and practice" Comput. Methods Appl. Mech. Engrg. 194 (2005) 39023933

[22] Melanie Mitchell "Introduction to genetic Algorithms" MIT Press, 978-262-63185-3

[23] A. B. H. Yedder "MyGa : un algorithme génétique écrit en Fortran" http://www .adel.online.fr/MyGa/

[24] M. Borchardt, private communication

[25] P. Merkel, "Solution of stellarator boundary value problems with external currents" Nucl. Fusion 27 No. 5 (1987) pp.867

[26] P. Merkel, "Applications of the Neumann problem to stellarators: Magnetic Surfaces, coils, free-boundary equilibrium, magnetic diagnostics" Theory of Fusion Plasmas, Workshop Proceedings, Varenna, Italy, Aug. 24-28 1987 (pp. 25-46)

[27] P. Helander Theory of plasma confinement in non-axisymmetric magnetic fields Rep. Progr. Phys. 77, 087001 (2014).

[28] V. V. Nemov et al., "Evaluation of $1 / \nu$ neoclassical transport in stellarators", Phys. Plasmas 6 No. 12 (1999) pp. 4622

[29] V. V. Nemov et al., "The $\nabla B$ drift velocity of trapped particles in stellarators", Phys. Plasmas 12 (2005) 112507

[30] R. H. Fowler, J. A. Rome, J, F. Lyon, "Monte Carlo studies of transport in stellarators" Phys. Fluids 28 (1), Jan 1985, pp. 338

[31] NCSX team, private communication

[32] V.D. Shafranov, L. E. Zakharov, "Use of the virtual casing principle in calculating the containing magnetic field in toroidal plasma systems" Nucl. Fusion 12 (1972) pp. 599

[33] J. D. Hanson, "The virtual casing principle and Helmholtz's theorem" Plasma Phys. Control. Fusion 57 (2015) 115006 
[34] P. Merkel, private communication

[35] C. D. Beidler et al., "The HELIAS Reactor" Proceedings of the 16th Int. Conference on Fusion Energy (Montreal, 1996), IAEA, Vienna (1997), Vol 3, p. 407-419

[36] H. L. Berk et al., "Existence and calculation of sharp boundary magnetohydrodynamic equilibrium in threedimensional toroidal geometry" Phys. Fluids 29 (1998) pp. 3281

[37] M. McGann, S. R. Hudson, R. L. Dewar, G. von Nessi, "Hamilton-Jacobi theory for continuation of magnetic field across a toroidal surface supporting a plasma pressure discontinuity" Physics Letters A 374 (2010) p. 33083314

[38] P. Merkel, M. Drevlak "Coils for 3d MHD equilibria" 1998 International Congress on Plasma Physics \& 25th EPS Conference on Controlled Fusion and Plasma Physics, Praha, June 29 - July 3, 1998, Ed. P. Pavlo, pp. 1745

[39] W. Lotz, P. Merkel, J. Nührenberg, E. Strumberger, "Collisionless alpha-particle confinement in stellarators" Plasma Phys. Control. Fusion 34 No. 6 (1992) pp. 1037

[40] D. J. Strickler, L. A. Berry, S. P. Hirshman, "Designing coils for compact stellarators" Fusion Science and Technology 41 No. 3 (2002) pp. 107

[41] Caoxiang Zhu et al., "New method to design stellarator coils without the winding surface” Nucl. Fusion 58 (2018) 016008

[42] S.R. Hudson et al. "Differentiating the shape of stellarator coils with respect to the plasma boundary" Physics Letters A 382 (2018) 27322737

[43] M. Landreman "An improved current potential method for fast computation of stellarator coil shapes" Nucl. Fusion 57 (2017) 046003

[44] A. H. Boozer, "Optimization of the current potential for stellarator coils", Phys. Plasmas Vol. 7 No. 2 (2000), pp. 629

[45] L.-P. Ku, A. H. Boozer, "Stellarator coil design and plasma sensitivity", Phys. Plasmas 17 (2010) 122503

[46] A. H. Boozer "Stellarator Design" J. Plasma Phys. (2015), Vol. 81, 515810606

[47] D. J. Strickler et al., "Integrated Plasma and Coil Optimization for Compact Stellarators", 19th IAEA Fusion Energy Conference, Lyon, France, Oct. 2002

[48] F. Brochard et al., "Physics and Engineering Design of ESTELL QuasiAxisymmetric Stellarator" 41st EPS Conference on Plasma Physics (2014) P2.081

[49] M. Drevlak et al., "ESTELL: A Quasi-Toroidally Symmetric Stellarator" Contrib. Plasma Phys. 53 No. 6 (2013) p. 459468 http://www.adel.online.fr/MyGa/

[50] C. D. Beidler et al., "Benchmarking of the mono-energetic transport Coefficients results from the International Collaboration on Neoclassical Transport in Stellarators (ICNTS)" Nuclear Fusion 51 (2011) 076001 